\title{
A historiography of the hyperlink: Periodizing the web through the changing role of the hyperlink
}

Anne Helmond, University of Amsterdam

\section{Introduction: Periodizing the web through the hyperlink}

In this chapter I provide a historiography of one of the core elements of the web, the hypertext link. I do so with the specific purpose of tracing the various roles of this central web object as a way to understand social, technical and commercial transformations of the web. That is, the hyperlink is positioned as a way to historicize larger web developments and as an alternative way to periodize the web.

The dominant way of web periodization is through what Allen calls a 'discourse of versions' (2013), established by Tim O'Reilly who labeled the period after the dot-com crash 'Web 2.0' (2005), a marketing term he used to claim this period as new and upgraded, whilst at the same time promising a sense of continuity (Allen, 2013: 5). In doing so, O'Reilly invented the idea of Web 1.0 in retrospect and created a perception of the web before, Web 1.0, and after, Web 2.0, the dot-com crash (Allen, 2013). Whilst O'Reilly stresses the continuities between the two periods and is careful to not position the shift as a technological upgrade, he nonetheless creates a juxtaposition by characterizing Web 1.0 as a web for 'publishing' and Web 2.0 as a web for 'participation' (2005). This supposed shift from Web 1.0 as the 'read-only' static 
Helmond A (2018) A Historiography of the Hyperlink: Periodizing the Web Through the Changing Role of the Hyperlink. In: Brügger N and Milligan I (eds) The SAGE Handbook of Web History. London, UK: SAGE Publications, pp. 227-241.

publishing web to Web 2.0 as the 'read-write' participatory social web has since become the web's dominant historical narrative (Song, 2010; Allen, 2013; Ankerson, 2015; Helmond, 2015; Stevenson, 2014).

Following Allen's interpretation and critique of this narrative, Megan Ankerson proposes to 'put aside the discourse of versions and approach the web and web historiography as a site of ongoing configuration' by employing Lucy Suchman's notion of 'configuration' as 'a device for studying how material artifacts and cultural imaginaries are joined together' and how these are connected to specific production practices (Ankerson, 2015: 3).

Similar to Ankerson's consideration of web historiography through forms of alignment and configuration, I approach my periodization of the web through one of the core infrastructural elements of the web, the hyperlink, by tracing its production and use practices within a view of the web as a complex social, techno-commercial configuration. Such focus does not aim to merely describe the technological progress of the hyperlink as a material artifact, but rather, to situate its development within the diverse practices of end-users, webmasters, bloggers, and developers within the context of the software, platforms, search engines, and apps that are involved in the creation, distribution and consumption of hyperlinks. At the same time, I consider the hyperlink's imagined capabilities by the actors involved in its production and distribution. Which social, political or economic purpose does the hyperlink serve for them? I argue that there is an ongoing -and sometimes conflicting-interpretation of what the hyperlink is 
or should be, and that there is a constant negotiation between specific production and use practices as well as an unfolding alignment with social and economic incentives.

In what follows, I argue that the hyperlink is not a timeless object and that it may be employed to analyze changes in the social, technical and commercial configuration of the web. I do so by providing a periodization of the hyperlink based on its historically diverse roles at six key moments (or episodes) that are characterized by distinct devices. I provide a history that discusses these six episodes individually whilst demonstrating their interconnections, thus highlighting how these episodes may run parallel. First, the proto-hyperlink as envisioned in early hypertext systems; second, the hypertext link as fabric of the web and navigational object in the early pre-search web; third, the hyperlink as the currency of the web in the heydays of the search engine era; fourth; the role of hyperlinks in building the blogosphere and the introduction of new link types; fifth, the effects of platformization on the hyperlink by social media platforms turning the link into an analytical device for data-harvesting; and sixth, the disappearance of the 'traditional' hypertext link as the prime connection mechanism with the rise of mobile apps.

\section{Tracing the hyperlink}

The history of the hyperlink as a reference and navigational object is often traced back to the footnote (De Maeyer, 2013), which functions as a way to explicitly link to additional information and source material within a text (Grafton, 1997), and to even older forms of inter-textual connections (Halavais, 2008; Brügger, 2017). In his 
genealogy of the hyperlink, Niels Brügger positions the hyperlink within the longer and broader history of links as mechanisms for interconnecting pieces of text (2017). He identifies three phases in his history of intertextual linking and observes how each period is characterized by a specific type of media: first, links in non-digital media such as clay tablets, printed books, and mechanical systems, second, links in stand-alone digital computers, and third, links in networked digital computers (2017). The history of the link presented here mainly focuses on the third period, which is characterized by the advent of interconnected computer systems, digital hypertext systems, and the rise of the World Wide Web. In particular I focus on the role of search engines, blog software, social media platforms, and apps in the evolution of the role of the hyperlink on the web and the mobile space.

On the web hyperlinks have evolved beyond citations to serve a wide range of social and technical functions (Halavais, 2008). Thus, instead of thinking about hypertext and hyperlinks as 'remediations' of older media types and linkeages (cf. Bolter, 1990), I follow Elmer's call that if we wish to understand links and linking on the web 'we must also come to grips with the specific architectural logic of the web' and consider the hyperlink within its web-native environment (2001). That is, I consider the medium-specificity of the hyperlink as a web-native object (Rogers, 2013; Helmond, 2013) by focusing on the infrastructure of the web and the actors involved in the production and distribution of hyperlinks. In what follows next, I discuss the changing purpose, use and function of the hyperlink over time in six episodes, each characterized by a number of human and non-human protagonists that have played a major role in the 
history of the hyperlink.

\section{Episode 1. The proto-hyperlink in early hypertext systems}

Most commonly, the lineage of the hypertext link is traced back to pre-web hypertext systems (Barnet, in this volume; Elmer, 2001), as a way to interconnect and make accessible our ephemeral human knowledge (Barnet, 2014). In her history of hypertext, Barnet (in this volume; 2014) describes different visions, models and implementations of hypertext systems before the web in order to emphasize that the web is just one but famous realization of a hypertext system. It is beyond the scope of this chapter to describe the development of these systems at length. The history of hypertext has been extensively discussed elsewhere by scholars from literature studies, game studies, information science, and new media studies (see Aarseth, 1997; Barnet in this volume; Barnet, 2014; Bolter, 1991; Brügger, 2017; Elmer, 2001; Halavais, 2008; Kirschenbaum, 2001; Krapp, 2006; Landow, 1991; Wardrip-Fruin, 2004; Wright, $2014 ;)$

However, it is important to briefly consider a number of proto-hypertext systems and the types of interconnections they conceived to understand links as part of technical infrastructures for organizing and distributing information. One of the earliest proto-hypertext systems is Paul Otlet's Mondothèque, a device for researchers, providing one of the many visions on how to organize the world's information (Rayward, 1994; Wright, 2014a; Wright, 2014b). Otlet aimed 'to promote universal access to human knowledge through a global information network that he dubbed the 
"Mundaneum"” (Wright, 2014a: 5). Knowledge was stored in and connected to the universal library of the Mundaneum via a personalized multimedia workstation called the 'Mondothèque' (Wright, 2014a; van den Heuvel \& Rayward, 2011). The Mondothèque, which remained an unrealized prototype, should be understood as 'more than just a platform for consuming information; it was an active tool for knowledge production' (Wright, 2014a: 235) enabling users to create links between various types of media documents and pieces of knowledge. Wright sees Otlet's Mondothèque as the forgotten European pre-cursor to Vannevar Bush's Memex, a proto-hypertext system in which (hyper)links were conceptualized as associational markers to establish 'trails' between documents (1945). The Memex aimed to organize, sort and structure the world's knowledge via associative indexing. However, what distinguishes the Otlet's from Bush' visions is that 'Otlet saw the network as essential to his vision of a worldwide platform for knowledge sharing; Bush envisioned the Memex as a stand-alone machine' (Wright, 2014b). Otlet and Bush' devices were analogue machines that relied on microfilm for the storage of data but both remained unbuilt prototypes. The advent of the (networked) computer introduced new ideas about organizing and connecting the world's information. Ted Nelson and Doug Engelbart were two pioneers working on early hypertext systems before the advent of the web.

The word 'hyperlink' has been attributed to Ted Nelson who worked on the first hypertext system, Project Xanadu, in the 1960s. The system was never entirely built but a number of partially functioning Xanadu prototypes were released since the late 1990s (Nelson, 2016). Nelson coined the word 'hypertext' (1965) in a paper outlining an 
evolutionary file structure for an automatic personal filing system, in which he quotes Bush' ideas about the Memex at length. Nelson used the word hypertext to describe how text within the system could be accessed and retrieved in a nonlinear fashion where readers could follow hypertext links to read additional information. A noteworthy core element of Nelson's Xanadu was the idea of unbreakable two-way links (1999: 1) as a way to implement 'transclusion', a type of interlinking of 'the same thing knowably and visibly in more than once place'.

Around the same time as Nelson was working on Xanadu, Doug Engelbart was creating the oN-Line System (NLS), inspired by Bush' Memex. NLS was a system for cross-referencing, based on the basic premise that you could link to anything and to anywhere through the Link function (Barnett, in this volume). The NLS system had fine-grained linking capabilities and links within the system represented a relationship (Barnett, in this volume). ${ }^{1}$ Both Xanadu and NLS were never adopted on a wide scale and it wasn't until the late 80s that Apple started selling computers with a pre-installed version of HyperCard, a piece of hypermedia software, that hypertext became available to the larger public and became 'de facto the archetype of what hypertext -and hyperlinking-was' (Brügger, 2017; Barnet, 2014: xxii). With the advent of the World Wide Web (the web), the ideas of hypertext and hyperlinking would be further exposed to a larger public.

\section{Episode 2. The hyperlink as fabric of the web}

In his book Weaving the Web on the history of the web, Tim Berners-Lee acknowledges 
Helmond A (2018) A Historiography of the Hyperlink: Periodizing the Web Through the Changing Role of the Hyperlink. In: Brügger N and Milligan I (eds) The SAGE Handbook of Web History. London, UK: SAGE Publications, pp. 227-241.

the ideas of Bush, Nelson and Engelbart in developing a hypertext system (2000: 5-6). He describes how his creation was also inspired by the organizing structures of the mind in terms of making and storing connections through (random) associations (2000: 10). Berners-Lee describes his task so as to 'marry' the development of the internet with early ideas of hypertext and hypertext links (2000: 6) to form 'a web of information' (2000: 4). His vision of the web was inspired by the following idea: 'Suppose all the information stored on computers everywhere were linked [...]. Suppose I could program my computer to create a space in which anything could be linked to anything. [...]' (2000: 4). To create such an interlinked information space, Berners-Lee created the Universal Resource Identifier (URI) to identify any object on the web, the HyperText Transfer Protocol (HTTP) for transferring hypertext, and the Hypertext Markup Language (HTML) as a common language or 'basic lingua franca' for writing and representing hypertext pages (2000: 36-40). Berners-Lee considered the URI the 'most fundamental innovation of the web' because the hypertext link contains the destination URI telling the browser where to find the document (2000: 37). HTML in its turn functions as 'the fabric' (Berners-Lee, Cailliau, Luotonen, Nielsen, \& Secret: 1994) or 'connective tissue' (Berners-Lee: 1998) of the web. One of the central elements of HTML is the hypertext link as 'the basic hypertext construct' enabling connections between different resources on the web (W3C, n.d.). Hypertext links, commonly referred to as hyperlinks, web links, or just simply links, allow the creation of interconnections between web sites, web pages, and other web objects. As such, they bear the capacity to weave disparate entities of the web together into 'a single, global information space', which Berners-Lee called the World Wide Web (2000: 4). 
Helmond A (2018) A Historiography of the Hyperlink: Periodizing the Web Through the Changing Role of the Hyperlink. In: Brügger N and Milligan I (eds) The SAGE Handbook of Web History. London, UK: SAGE Publications, pp. 227-241.

Universality was key to the design of the web as '[a] hypertext link must be able to link to anything' (107) and anyone should be able to create links. Berners-Lee considered 'the right to link' as 'the very basic building unit for the whole Web' (139). Similar to, but distinct from, Nelson's implementation of the two-way link, Berners-Lee contemplated designing the web with bidirectional links (Berners-Lee, n.d.) but these were never implemented as part of an architectural decision because mono-directional links enabled the web to scale quickly (Berners-Lee, 1998). Another important aspect in the design of the link was that whilst Berners-Lee saw that it could be used as 'a way of transmitting judgements of quality', he made clear that 'the intention in the design of the web was that normal links should simply be references, with no implied meaning' (1997) and that the hyperlink does not imply any type of endorsement (2000: 139).

Key to the initial popularization of hyperlinks was the introduction of graphical browsers such as Mosaic by indicating clickable hyperlinks in blue-colored underlined text which became the de facto standard for displaying links on the web (Weinreich, Obendorf, \& Lamersdorf, 2001). Whilst anyone could potentially create hyperlinks by learning HTML, in these early days of the web this act was reserved to webmasters who could make and run their own websites. With the advent of HTML-editors, content management systems, blog software and free website services such as Tripod and Geocities, it became easier to create websites and to link to other sites. This period of mass amateur website building, which Olia Lialina refers to as the 'vernacular web', was driven by a do-it-yourself ethos with very few design rules-except for valid HTML- and led to an explosion of websites with an amateur aesthetic (2009: 27). 
According to Lialina this web was 'fascinated by the power of links' and 'people felt it was their responsibility to configure the environment and build the infrastructure' by linking to other pages and by listing external link lists on their websites (27). In addition to building the web's infrastructure, people also built communities through links by engaging in 'web rings', a way of interlinking websites with similar topics (Lialina, 2009: 27; Elmer, 1999). These webrings also contributed to the discoverability of new websites and offered a subject-based index of a set of websites (Elmer, 1999).

With the growth of the web it became more and more difficult to find sites interesting and relevant websites. This led to the creation of new services that aimed to index the web, with search engines like Aliweb, Excite and AltaVista (xx, in this volume), to provide an expert directory of links, ${ }^{2}$ like DMOZ and the Yahoo! Directory, or to serve as the entry point of the web by providing professionally curated links, with portals like MyNetscape and My Yahoo!. These mostly new commercial actors made money off hyperlinks through the strategic placement of links or 'featured links' on their websites (Hargittai, 2000; Rogers, 2002). Thus, hyperlinks have given rise to an industry of new commercial actors on the web such as content aggregators, web portals, and search engines (cf. Dellarocas, Katona, \& Rand, 2013: 2360). A new company, Google, would turn hyperlinks and the interlinked infrastructure of the web into a very successful business model.

\section{Episode 3. The hyperlink as currency of the web}

An important moment in the history of both the web and the hyperlink has been the 
introduction and rise of search engines. Here, I focus on the role of Google as the search engine that 'built its empire from an appreciation of the Net's underlying architecture', as it conceived of a 'web/info-centric business model that was built upon the harvesting and crawling of hyperlinks' (Elmer, 2006: 9). What distinguished Google from previous search engines is that it employed hyperlinks as a way to calculate the relevance and reputation of a site, by considering -amongst other factors- who links to whom and how often a site is linked to (Brin and Page, 1998). Google's PageRank algorithm calculates the relevance of a website based on the quality or importance of other websites linking to it (Brin and Page, 1998) and as a result not all hyperlinks have equal value for Google. Whilst Google currently takes over 200 signals into account, PageRank is still an important factor to determine a site's relevance (Search Console Help, 2017).

Google's 'industrialization' of the hyperlink (Turow, 2008: 3) has created the so-called 'link economy' (Walker, 2002; Rogers, 2002) in which search engines determine the value of links and where links are exchanged, bought or sold by webmasters and spammers. Google turned the link from a navigational object into 'the currency of the web' by interpreting links as 'objective, democratic and machine-readable signs of value' (Walker, 2002: 72). Google's business model is built on the 'open web', a web built on open standards and where links can be followed from one page to another and indexed by search engines. The foregrounding of the link as a sign of value and medium of exchange raises questions about the actors involved in the production, indexation and distribution of links. In this sense, the hyperlink provides a way to understand the early commercialization of the web, which has traditionally focused on businesses 'rushing' 
to the web to build websites as a form of presence (cf. Rogers, 2002), but instead focuses on the political economy of the link. Such a perspective considers its role as a currency (Walker, 2002) and draws attention to the rise of new web-specific services that monetize on the hyperlink as the core feature of the web, and the rise of new linking practices and strategies. With the new role of the link as the currency of the web, actors involved in creating hyperlinks became aware of their strategic value leading to a whole new industry of manipulation around search engines to achieve higher rankings (Halavais, 2008: 49-50). Next, I elaborate on the political economy of linking and the constant negotiation between those creating links and those indexing and distributing links by focusing on the role of links and the practices of linking in the blogosphere and the creation of new types of links.

\section{Episode 4. Building the blogosphere and the introduction of new link types}

Besides directories, web portals, web rings, and search engines trying to organize the web in the late 1990 s, there were also very important individual actors such as webmasters and bloggers who would point other users to interesting websites and pages through link lists on their websites and blogs. Blogs are often seen as a specific genre of websites, ${ }^{3}$ defined as by their form as 'frequently updated, reverse-chronological entries on a single Web page' (Blood, 2004: 53). These entries called 'posts' constitute the main units or building blocks of the blog, in contrast to the 'page' unit of the website. In order to be able to link to a single post, instead of a whole page, blogger Jason Kottke started implementing permanent URLs for each entry on his blog (2000). This idea was quickly taken up by developers from blog software company Blogger who implemented 
Helmond A (2018) A Historiography of the Hyperlink: Periodizing the Web Through the Changing Role of the Hyperlink. In: Brügger N and Milligan I (eds) The SAGE Handbook of Web History. London, UK: SAGE Publications, pp. 227-241.

'permalinks' into their software to give 'each blog entry a permanent location-a distinct URL-at which it could be referenced' (Blood, 2004: 54). This new link type was quickly adopted by other bloggers and blog software developers and the permalink became 'a canonical component of the standard Weblog entry' (Blood, 2004: 54).

Early blogger Rebecca Blood argues that the 'original weblogs were link-driven sites' (2000) and that the prototypical blog focuses on linking to other blogs to provide commentary on interesting blog posts (2000; Herring et al., 2005). But links did not only play an important role within a blog entry, bloggers also often created 'blogrolls' to link to other interesting blogs in the sidebar of their blogs. Al these links between blogs create an interconnected network of blogs called 'the blogosphere', although the degree its interconnectedness might be overestimated (Herring et al., 2005). The ritual practices of linking stabilized the blog as a web format and created a sense of community as bloggers would start referring to themselves as the 'weblog community' (Siles, 2011: 745; Ammann, 2009).

Before the advent of blog software, which originated and gained popularity between 1997-1999, bloggers would manually create hyperlinks to link to other blogs (Blood, 2000; Helmond, 2008). The practice of blogging and linking was made easier with the implementation of linking features into blog software and What You See Is What You Get (WYSIWYG)-editors (Blood, 2004: 54). Hereafter, bloggers would only have to insert a URL and the blog software would automatically generate the corresponding HTML code for the hyperlink. Blog software also enabled automated interlinking 
Helmond A (2018) A Historiography of the Hyperlink: Periodizing the Web Through the Changing Role of the Hyperlink. In: Brügger N and Milligan I (eds) The SAGE Handbook of Web History. London, UK: SAGE Publications, pp. 227-241.

between blogs with the creation of two new link types that were built on top of the permalink: the trackback and pingback. Blog software can 'ping' or notify other blog software of an incoming link by automatically 'placing a reciprocal link-a trackback - in the entry they have just referenced' (Blood, 2004: 55). Trackbacks are a semi-manual type of notification and interlinking system since bloggers have to manually send the trackback notification from within the software's interface. Receiving trackbacks could be automated since blog software can be configured to automatically receive and place trackbacks underneath a post. Unfortunately, trackbacks turned out to be very prone to spam since the receiving software did not not verify the incoming link and incoming links could therefore very easily be faked. Spammers used trackbacks to artificially boost the ranking of their sites in search engines and pingbacks were developed to partially solve the problems of trackbacks. Pingbacks automatically send notifications to other blogs and the receiving blog software verifies the incoming link. Both trackbacks and pingbacks (semi-)automatically interlink blogs and render the links between blogs visible on both ends, resembling the idea of a two-way link. Trackbacks and pingbacks draw attention to the changing configuration of actors involved in the production and distribution of links. Blog software introduced and standardized new types of links such as the permalink, the trackback and pingback, and became an active agent in creating links between blogs and the interlinking of blogs into a blogosphere (Helmond, 2008; Weltevrede \& Helmond, 2012; Helmond, 2013).

Another important blog feature that highlights the constant negotiations taking place between various actors involved in creating, distributing, and exploiting links is the blog 
Helmond A (2018) A Historiography of the Hyperlink: Periodizing the Web Through the Changing Role of the Hyperlink. In: Brügger N and Milligan I (eds) The SAGE Handbook of Web History. London, UK: SAGE Publications, pp. 227-241.

comment. Whilst previously the act of linking was mainly reserved to webmasters and bloggers, ${ }^{4}$ blog comments underneath blog posts opened up the blog to links from blog visitors. This form of user-generated linking expanded the participatory ideals of blogging as a way 'to democratize publishing' (Blood, 2004: 55) by also giving blog readers a voice. However, opening up the comment space made blogs even more prone to spam. Spammers would not only target the trackback mechanism but also the comment space with links to boost their own rankings. This time an attempt to create a solution to combat link spam was developed by Google, the key actor in the link economy, who suffered from spam in its search engine results as a result of the new linking capabilities. On January 18, 2005, Google announced its measure to prevent comment spam, a new hyperlink attribute called 'nofollow'. In HTML, elements can have parameters called attributes where the $<a>$ element defines the hypertext link, the 'href' attribute defines the destination of a link, and the 'rel' attribute specifies 'the relationship between the document containing the hyperlink and the destination resource' (W3C, 2014). Google introduced the 'nofollow' value as part of the hyperlink's 'rel' attribute to indicate a particular type of relationship between the source and destination link: 'From now on, when Google sees the attribute (rel="nofollow") on hyperlinks, those links won't get any credit when we rank websites in our search results' (Cutts \& Shellen, 2005). No credit meant no value for a website's PageRank, severely diminishing the intended goal of the spammers. The nofollow attribute was introduced in collaboration with a number of partners, including major blog software developers such as Blogger and WordPress, who implemented it into their software so that every link in a blog comment would automatically receive the nofollow attribute. The effect of 
nofollow is that in Google's world, not all links have equal value, since links in comments with the nofollow attribute do not pass on value for a site's PageRank. The role of 'nofollow' to prevent spam is still a pressing issue today, as Google regularly warns webmasters to follow their linking guidelines and to use 'nofollow' where appropriate or else they may see their sites' rankings penalized (Google Webspam Team, 2017).

\section{Episode 5. The effects of platformization on the hyperlink}

Whilst the hyperlink has technically not changed since its inception, in the previous episodes I have demonstrated how search engines and blog software have appropriated or have added features to the hyperlink for their own purposes. This next episode discusses the implications of the 'platformization' of the web (Helmond 2015), a term used to refer to the rise of social media platforms and the consequences of their extension into the web, for the hyperlink. It provides a way to conceptualize how social media platforms have imposed their own infrastructural and economic model onto the web by repurposing web-native objects, such as the hyperlink, for their own gain. I discuss the consequences of the rise of social media for the hyperlink by addressing three issues: first, the further automation of linking through social buttons, second, the reconfiguration of the hyperlink through social buttons to fit the underlying business models of the associated social media platforms, and third, the increasing invisibility of the hyperlink in social media.

With the introduction of social bookmarking icons, now commonly known as social 
Helmond A (2018) A Historiography of the Hyperlink: Periodizing the Web Through the Changing Role of the Hyperlink. In: Brügger N and Milligan I (eds) The SAGE Handbook of Web History. London, UK: SAGE Publications, pp. 227-241.

buttons, social media platforms have developed ways to facilitate easy link sharing across platforms (Helmond, 2013). ${ }^{5}$ Sharing a website article using a social button such as Facebook's Share button automatically creates a status update displaying a preview of the link's content: the article's title, subtitle, image, domain name and author. ${ }^{6}$ The status update with the link preview does not show the linked URL but instead is reduced to the URL's domain name which then links to the individual article. Sharing an article directly on Facebook is handled slightly different since posting a link shows both the full URL in the status update and creates a link preview underneath the post. The automation of link sharing via social buttons creates a two-way link:

Facebook also employs linking mechanisms that closely resemble the bidirectional links proposed by Bush, Nelson, and so on. Whereas a typical Web hyperlink usually goes in one direction-from source document to target hyperlink-links on Facebook typically work both ways; a comment or "like" interaction will show up in multiple threads; and a user may follow a link back to see the original commenter's page (Wright, 2014: 290)

However, Facebook does not support traditional linking practices of being able to create a hyperlink by linking a word in a Facebook post to a specific URL destination on the web. The only 'links' you can create are to internal Facebook items such as users and pages (Berners-Lee, 2010). Software developer Dave Winer is a strong advocate of implementing hyperlinking into Facebook. The current implementation, he argues, is not a form of linking and is 'really hurting the rest of the web' by not allowing to freely link to other places on the web (2016). Tim Berners-Lee has also expressed his concerns in regard to websites and web services that develop their own linking mechanisms that 
Helmond A (2018) A Historiography of the Hyperlink: Periodizing the Web Through the Changing Role of the Hyperlink. In: Brügger N and Milligan I (eds) The SAGE Handbook of Web History. London, UK: SAGE Publications, pp. 227-241.

do not interoperate with the web's open standards and formats (2010). He argues that they are undermining the web's principle of universality meaning that you can link to anything, where 'the URI is the key to universality' (2010). He explains how social media platforms are becoming silos by walling off their information: 'The isolation occurs because each piece of information does not have a URI. Connections among data exist only within a site.' Berners-Lee warns that ' $[t]$ he more this kind of architecture gains widespread use, the more the Web becomes fragmented, and the less we enjoy a single, universal information space' (2010: 82). Links are essential in weaving the web into a single place and social media platforms are prohibiting a specific type of linking practice, the traditional hyperlink, over their own forms of interlinking. In addition, many social media platforms also reconfigure links posted to their platforms so that they fit the underlying platform architectures and business models. They do so by turning links shared to their platforms into shortened URLs, a new type of link which gained popularity on Twitter.

On Twitter, users can only use up to 140 characters for their tweets which means that posting a link will take up a significant amount of valuable characters. Users turned to URL shorteners such as TinyURL to shorten their long links into shortened links, e.g. http://tinyurl.com/newlink. In June 2011 Twitter built its own URL shortener t.co into its platform architecture and from that moment onwards every link posted to Twitter is automatically shortened into a t.co URL. ${ }^{7}$ Twitter hides this transformation of the hyperlink from its users, by displaying an abbreviated version of the original long link in the front-end, the platform's user interface as visible in the browser, whilst using the 
actual shortened link for a variety of different purposes in the back-end, the platform's data infrastructure. ${ }^{8}$ By routing all links through its platform, Twitter can not only detect and filter out spam links but it can also collect valuable link data. Twitter can now easily track how many times a link has been shared, clicked on, by whom and when, on its platform. But it can also gather many of these statistics when the link is distributed outside of its platform boundaries. As I have argued elsewhere, platforms have turned the link into an analytical device by repurposing it into a data-rich shortened URL:

By automatically wrapping links in tweets with a t.co URL, Twitter makes this shared data on its platform 'algorithm ready' by reconfiguring the hyperlink to fit the platform. The automatic processing of the hyperlink and its reconfiguration into an analytical device in order to become part of an algorithmic system is what I refer to as the algorithmization of the hyperlink (Helmond 2013).

On social media platforms, the link no longer just functions as a navigational device where a link in a tweet points to another location on the web, but it also provides valuable data such as link statistics. This data serves as input for the platform's various algorithms that determine relevant and trending content but is also used by Twitter's marketing partners for their own commercial purposes such as advertising (Helmond, 2013). Platforms have appropriated the link as an object to measure web activities, attention and other forms of engagement to fit their own economic agendas.

Next, I briefly turn to the current state of web development to reflect on the decline of the hyperlink and the introduction of new link types such as mobile deep links and app 
links with the rise of mobile apps.

\section{Episode 6. Beyond the web: Mobile apps and deep linking}

The previous sections have argued that in order to understand the evolution of the hyperlink and associated linking practices we should consider the actors involved in the production and reconfiguration of the hyperlink. In the changing political economy of linking a variety of actors has been involved over time: from webmasters, bloggers and social media users to search engines, blog software, and social media platforms, to only name a few. I have argued that in order to understand what the hyperlink is, can do, or is made to do, we should take consider it role within the transforming socio-techno-commercial environment of the web. When considering the present role of the hyperlink, its evolution and implementation should also be considered beyond the web. That is, with the introduction of mobile phones and the increasing ubiquity of mobile network data, in recent years we have seen the development of the mobile web (xx, in this volume) with pages specifically built for or adjusted to smaller screens and lower bandwidth. However, instead of building mobile web pages or services many companies have built dedicated mobile apps. For many, apps have become the main entry point to the web and internet-based platforms (comScore, 2016). Whilst the mobile web is based on the same standards as the web, and links between pages are created using hyperlinks, apps employ proprietary linking mechanisms. Instead, apps employ so-called mobile or app 'deep links' to enable app interlinking but these links are not based on open standards. In his defense of the open web, Berners-Lee describes how deep linking in apps works and the consequences of this type of linking: 'Apple's 
Helmond A (2018) A Historiography of the Hyperlink: Periodizing the Web Through the Changing Role of the Hyperlink. In: Brügger N and Milligan I (eds) The SAGE Handbook of Web History. London, UK: SAGE Publications, pp. 227-241.

iTune system, for example, identifies songs and videos using URIs that are open. But instead of "http:" the addresses begin with "itunes:," which is proprietary. You can access an “itunes:” link only using Apple's proprietary iTunes program' (2010: 83). Apps do not employ open link standards which, similar to the practices of social media platforms, turns them into stand-alone objects or little unlinkable islands that often function separate from the web thereby turning them into 'closed worlds' or centralized 'walled gardens' (Berners-Lee, 2010: 83). To create interconnections between apps and the web, companies such as Apple, Google and Facebook have introduced new link types for linking between apps and for linking to specific content within apps. Facebook launched App Links at its F8 Developer Conference in 2014 as 'an open cross platform solution for deep linking to content in your mobile app' (Facebook for Developers, n.d.). For Facebook, these App Links also provide a way to gather app analytics such as 'traffic and usage information' for who is using your app (Facebook for Developers, n.d.) thereby positioning App Links both als navigational and analytical devices. In May 2015 Google announced its own 'App links, along with App Indexing for Google search' (Eason, 2015). A month later, during the WWDC developer conference, Apple also announced their own version, entitled 'Universal Links,' (Apple Developer, n.d.-a) seemingly referring to the universality principle of hyperlinks on the web. Similar to websites and web pages which are linked or 'woven' together by hyperlinks to create the World Wide Web, Apple explicates how 'an app exists as part of an ecosystem' of other apps (Apple Developer, n.d.-b). Universal Links are Apple's way of linking apps into an ecosystem similar to but separate from the web. Whilst these current developments may aim to address the current barrier of app-interlinking they are not 
providing a universal solution. ${ }^{9}$ With the introduction of deep links, Facebook's App

Links, Google's app links, and Apple's Universal Links these companies have contributed their own -mostly proprietary-solutions for app linking (and indexing) and have created a proliferation of new app link standards outside of the scope of $\mathrm{W} 3 \mathrm{C}$, which oversees open web standards such as HTML and the hypertext link. In addition, deep links do not only provide an app-native way of linking, but also app-native way to track users, and to collect valuable information for advertisers. At the same time this new link type points to the diminishing role of the hyperlink in the app space as a universal interconnector.

\section{Conclusion}

The hyperlink as one of the core infrastructural elements of the web has a long and rich history. Not only if we consider its potential precursors of intertextual links and its early employment in proto-hypertext systems, but also, and especially, its implementation on the web. In this chapter I have provided a historiography of the hyperlink by narrating its role in six episodes in the history of the web. These episodes demonstrate how the hyperlink has evolved from a feature to interlink information within technical knowledge infrastructures such as the Mondotheque, the Memex, Xanadu and the World Wide Web, to a feature that may be used to collect valuable information about the interlinked structure of the web as well as about the people clicking and viewing those links. That is, the hyperlink's role has shifted from functioning primarily as a navigational tool into an analytical device for data-harvesting. As such, the evolution of the hyperlink provides an important entry point into understanding the increasing 
commercialization of the web. The way in which the hyperlink has been employed socially by users and economically by search engines and social media platforms has changed significantly. By tracing how these actors have handled and appropriated the hyperlink over time we gain insights into its changing role and function on the web and in the app space.

The six episodes focused on the relation between the main actors involved in the production, distribution, and valorization of hyperlinks. These protagonists included human actors, such as webmasters, bloggers, blog software developers, technology entrepreneurs as well as non-human actors such as blog software, search engines, and social media platforms, and the companies behind them such as Google, Facebook, Twitter, and Apple. Each of these actors comes with its own very specific ideas, agendas or economic incentives of what the hyperlink is, could or should be. That is, there is a constant interplay between the material object of the hyperlink and the various actors involved in its production, distribution, and valorization. Instead of providing a lineage or teleological account of the hyperlink, the six episodes focused on the changing constellations of actors and how they employ the hyperlink for their own social, technical and economic incentives. The episodes demonstrate a number of continuities and discontinuities in the role of the hyperlink. First, the hyperlink is still a core element of the web and a central mechanism for creating and navigating connections between web pages and sites. However, these links are no longer manually created by webmasters, bloggers, and other types of web users, but linking has become increasingly (semi-)automated with the introduction of blog software and social buttons. 
As a consequence, the way in which the web becomes interconnected has changed.

Second, social media platforms and companies involved in app development have created their own interconnection and interlinking mechanisms, their own types of links, which function similar to, but are different from the traditional hyperlink. As a result, social media platforms and in particular apps are seen as walled gardens which operate their own logic of connection, largely disconnected from the World Wide Web at large. Custom linking mechanisms are not only developed for creating connections, but especially for tracing and tracking the movement of users and their data through social media platforms and apps.

By focusing on the political economy of linking over time, I have drawn attention to the increasing commercialization of the hyperlink. The hyperlink has seemingly lost its innocence since it is no longer just a way of navigating the web, but has become an object that can be monetized and that can employed to track users. Linking is no longer just 'weaving the web' but contributing to an infrastructure of valuation.

\section{Acknowledgements}

The author wishes to thank Fernando van der Vlist, the reviewer, and the editors for their for their valuable comments on this chapter. 


\section{References}

Aarseth, E. J. (1997). Cybertext: Perspectives on Ergodic Literature (First Edition edition). Baltimore, Md: Johns Hopkins University Press.

Allen, M. (2013). What was Web 2.0? Versions as the dominant mode of internet history. New Media \& Society, 15(2), 260-275. https://doi.org/10.1177/1461444812451567

Ammann, R. (2009, November 5). Blogosphere 1998: Analysis. Retrieved from http://tawawa.org/ark/2009/11/5/blogosphere-1998-analysis.html

Ankerson, M. S. (2015). Social Media and the "Read-Only" Web: Reconfiguring Social Logics and Historical Boundaries. Social Media + Society, 1(2), 2056305115621935. https://doi.org/10.1177/2056305115621935

Apple Developer. (n.d.-a). Seamless Linking to Your App. Retrieved March 4, 2017, from https://developer.apple.com/videos/play/wwdc2015/509/

Apple Developer. (n.d.-b). Universal Links for Developers. Retrieved March 4, 2017, from https://developer.apple.com/ios/universal-links/

Barnet, B. (2018). Hypertext Before the Web - or, What the Web Could Have Been. In N. Brügger \& I. Milligan (Eds.), Sage Handbook of Web History. London: Sage.

Barnet, B. (2014). Memory Machines: The Evolution of Hypertext. London: Anthem Press. 
Helmond A (2018) A Historiography of the Hyperlink: Periodizing the Web Through the Changing Role of the Hyperlink. In: Brügger N and Milligan I (eds) The SAGE Handbook of Web History. London, UK: SAGE Publications, pp. 227-241.

Berners-Lee, T. (1997, April). Links and Law. Retrieved from http://www.w3.org/DesignIssues/LinkLaw.html

Berners-Lee, T. (1998, September). Web Architecture from 50,000 feet. Retrieved February 17, 2017, from https://www.w3.org/DesignIssues/Architecture.html Berners-Lee, T. (2010, December). Long Live the Web: A Call for Continued Open Standards and Neutrality. Scientific American, (December 2010). Retrieved from http://www.scientificamerican.com/article/long-live-the-web/

Berners-Lee, T. (n.d.). Topology. Retrieved February 17, 2017, from https://www.w3.org/DesignIssues/Topology.html

Berners-Lee, T., Cailliau, R., Luotonen, A., Nielsen, H. F., \& Secret, A. (1994). The World-Wide Web. Communications of the ACM, 37(8), 76-82. https://doi.org/10.1145/179606.179671

Blood, R. (2000, September 7). Weblogs: a history and perspective. Retrieved from http://www.rebeccablood.net/essays/weblog_history.html

Blood, R. (2004). How blogging software reshapes the online community. Communications of the ACM, 47(12), 53-55. Retrieved from http://dl.acm.org/citation.cfm?id=1035165

Bolter, J. D. (1990). Writing Space: the Computer, Hypertext, and the History of Writing. Hillsdale, N.J: Routledge.

boyd, danah. (2006). A blogger's blog: Exploring the definition of a medium. 
Helmond A (2018) A Historiography of the Hyperlink: Periodizing the Web Through the Changing Role of the Hyperlink. In: Brügger N and Milligan I (eds) The SAGE Handbook of Web History. London, UK: SAGE Publications, pp. 227-241.

Reconstruction, 6(4). Retrieved from

http://www.danah.org/papers/ABloggersBlog.pdf

Brin, S., \& Page, L. (1998). The anatomy of a large-scale hypertextual Web search engine. Computer Networks and ISDN Systems, 30(1), 107-117. Retrieved from http://www.sciencedirect.com/science/article/pii/S016975529800110X

Brügger, N. (2017). The Web hyperlink: An outline of a genealogy. In N. Brügger (Ed.), Web 25: Histories from the first 25 Years of the World Wide Web. New York: Peter Lang Publishing.

Bush, V. (1945, July). As we may think. The Atlantic. Retrieved from http://www.theatlantic.com/magazine/archive/1945/07/as-we-may-think/303881/

Cutts, M., \& Shellen, J. (2005, January 19). Preventing comment spam. Retrieved October 29, 2012, from http://googleblog.blogspot.nl/2005/01/preventing-comment-spam.html

De Maeyer, J. (2013). Towards a hyperlinked society: A critical review of link studies. New Media \& Society, 15(5), 737-751. https://doi.org/10.1177/1461444812462851

Dellarocas, C., Katona, Z., \& Rand, W. (2013). Media, aggregators, and the link economy: Strategic hyperlink formation in content networks. Management Science, 59(10), 2360-2379. Retrieved from http://pubsonline.informs.org/doi/abs/10.1287/mnsc.2013.1710 
Eason, J. (2015, May 28). Android M Developer Preview \& Tools. Retrieved from https://android-developers.googleblog.com/2015/05/android-m-developer-previe w-tools.html

Elmer, G. (1999, January). Web Rings as Computer-Mediated Communication. CMC Magazine, (January). Retrieved from http://www.december.com/cmc/mag/1999/jan/elmer.html

Elmer, G. (2001). Hypertext on the Web: The Beginnings and Ends of Web Path-ology. Space and Culture, 10, 1-14.

Elmer, G. (2006). Re-tooling the Network Parsing the Links and Codes of the Web World. Convergence: The International Journal of Research into New Media Technologies, 12(1), 9-19. https://doi.org/10.1177/1354856506061549

Facebook for Developers. (n.d.). App Links - Documentation. Retrieved February 14, 2017, from https://developers.facebook.com/docs/applinks/analytics

Grafton, A. (1997). The Footnote: A Curious History. Cambridge, MA: Harvard University Press.

Google Webspam Team. (2017). A reminder about links in large-scale article campaigns. Retrieved May 26, 2017, from https://webmasters.googleblog.com/2017/05/a-reminder-about-links-in-large-scale. html

Halavais, A. (2008). The hyperlink as organizing principle. In J. Turow \& L. Tsui 
(Eds.), The hyperlinked society (pp. 39-55). Ann Arbor, MI: The University of Michigan Press.

Hargittai, E. (2000). Open portals or closed gates? Channeling content on the World Wide Web. Poetics, 27(4), 233-253. Retrieved from http://www.sciencedirect.com/science/article/pii/S0304422X00000061

Helmond, A. (2008). Blogging for engines. Blogs under the influence of software-engine relations. University of Amsterdam, Amsterdam. Retrieved from http://www.annehelmond.nl/2008/09/23/blogging-for-engines-blogs-under-the-i nfluence-of-software-engine-relations/

Helmond, A. (2013). The Algorithmization of the Hyperlink. Computational Culture, (3). Retrieved from http://computationalculture.net/article/the-algorithmization-of-the-hyperlink

Helmond, A. (2015). The Platformization of the Web: Making Web Data Platform Ready. Social Media + Society, 1(2). https://doi.org/10.1177/2056305115603080

Herring, S. C., Kouper, I., Paolillo, J. C., Scheidt, L. A., Tyworth, M., Welsch, P., ... Yu, N. (2005). Conversations in the blogosphere: An analysis“ from the bottom up.” In System Sciences, 2005. HICSS'05. Proceedings of the 38th Annual Hawaii International Conference on (p. 107b-107b). IEEE. Retrieved from http://ieeexplore.ieee.org/xpls/abs_all.jsp?arnumber=1385453 
Kirschenbaum, M. (2001). Materiality and matter and stuff: what electronic texts are made of. Electronic Book Review, 3. Retrieved from http://www.altx.com/ebr/riposte/rip12/rip12kir.htm

Kottke, J. (2000, March 5). Finally. Did you notice the. Retrieved March 3, 2017, from http://kottke.org/00/03/finally-did-you-notice-the

Krapp, P. (2006). Hypertext avant la lettre. In W. Chun \& T. Keenan (Eds.), New media, old media : a history and theory reader (pp. 359-374). New York: Routledge. Retrieved from https://www.researchgate.net/publication/259503242_Hypertext_avant_la_lettre

Landow, P. G. P. (Ed.). (1991). Hypertext: The Convergence of Contemporary Critical Theory and Technology. Baltimore: The Johns Hopkins University Press.

Lialina, O. (2009). A Vernacular Web. In O. Lialina \& D. Espenschied (Eds.), Digital Folklore (pp. 19-35). Stuttgart: Merz Akademie.

Nelson, T. H. (1965). Complex Information Processing: A File Structure for the Complex, the Changing and the Indeterminate. In Proceedings of the 1965 20th National Conference (pp. 84-100). New York, NY, USA: ACM. https://doi.org/10.1145/800197.806036

Nelson, T. H. (1999). Xanalogical Structure, Needed Now More Than Ever: Parallel Documents, Deep Links to Content, Deep Versioning, and Deep Re-use. $A C M$ Comput. Surv., 31(4es). https://doi.org/10.1145/345966.346033 
Helmond A (2018) A Historiography of the Hyperlink: Periodizing the Web Through the Changing Role of the Hyperlink. In: Brügger N and Milligan I (eds) The SAGE Handbook of Web History. London, UK: SAGE Publications, pp. 227-241.

Nelson, T. H. (2016, October 15). THE XANADU® PARALLEL UNIVERSE. Retrieved March 2, 2017, from http://xanadu.com/xUniverse-D6

O'Reilly, T. (2005, September 30). What is Web 2.0: Design patterns and business models for the next generation of software. Retrieved November 4, 2012, from http://oreilly.com/web2/archive/what-is-web-20.html

Rayward, W. B. (1994). Visions of Xanadu: Paul Otlet (1868-1944) and hypertext. Journal of the American Society for Information Science (1986-1998), 45(4), 235.

Rogers, R. (2002). Operating issue networks on the Web. Science as Culture, 11(2), 191-213. Retrieved from http://www.tandfonline.com/doi/abs/10.1080/09505430220137243

Rogers, R. (2013). Digital Methods. Cambridge, MA: The MIT Press.

Search Console Help. (n.d.). How Google Search Works. Retrieved May 26, 2017, from https://support.google.com/webmasters/answer/70897?hl=en

Siles, I. (2011). From online filter to web format: Articulating materiality and meaning in the early history of blogs. Social Studies of Science, 41(5), 737-758. Retrieved from http://sss.sagepub.com/content/41/5/737.short

Song, F. W. (2010). Theorizing web 2.0: A cultural perspective. Information, Communication \& Society, 13(2), 249-275. Retrieved from http://www.tandfonline.com/doi/abs/10.1080/13691180902914610 
Stevenson, M. (2014). Rethinking the participatory web: A history of HotWired's “new publishing paradigm,” 1994-1997. New Media \& Society, 1461444814555950. https://doi.org/10.1177/1461444814555950

Turow, J. (2008). Introduction: On Not Taking the Hyperlink for Granted. In J. Turow \& L. Tsui (Eds.), The Hyperlinked Society: Questioning Connections in the Digital Age. Ann Arbor, MI: University of Michigan Press.

Twitter. (2011, June 7). Link sharing made simple. Retrieved from https://blog.twitter.com/2011/link-sharing-made-simple

van den Heuvel, C., \& Rayward, W. B. (2011). Mondothèque. A multimedia desk in a global internet. Poster for the 7th Iteration on "Science Maps as Visual Interfaces to Digital Libraries” of the Places\&Spaces Mapping Science Exhibition.

W3C. (2014, October 28). 4.2.4 The link element. Retrieved March 2, 2017, from https://www.w3.org/TR/html5/document-metadata.html

W3C. (n.d.). Links. Retrieved July 17, 2013, from https://www.w3.org/TR/html401/struct/links.html

Walker, J. (2002). Links and power: the political economy of linking on the Web. In Proceedings of the thirteenth ACM conference on Hypertext and hypermedia (pp. 72-73). Retrieved from http://dl.acm.org/citation.cfm?id=513358

Wardrip-Fruin, N. (2004). What hypertext is. In Proceedings of the fifteenth ACM 
conference on Hypertext and hypermedia (pp. 126-127). ACM. Retrieved from http://dl.acm.org/citation.cfm?id=1012844

Weinreich, H., Obendorf, H., \& Lamersdorf, W. (2001). The Look of the Link Concepts for the User Interface of Extended Hyperlinks. In Proceedings of the 12th ACM Conference on Hypertext and Hypermedia (pp. 19-28). New York, NY, USA: ACM. https://doi.org/10.1145/504216.504225

Weltevrede, E., \& Helmond, A. (2012). Where do bloggers blog? Platform transitions within the historical Dutch blogosphere. First Monday, 17(2). https://doi.org/10.5210/fm.v17i2.3775

Winer, D. (2016, January 3). Facebook and linking is a big deal. Retrieved from http://scripting.com/liveblog/users/davewiner/2016/01/03/0783.html

Wright, A. (2014a). Cataloging the World: Paul Otlet and the Birth of the Information Age. Oxford: Oxford University Press.

Wright, A. (2014b, May 22). The Secret History of Hypertext. The Atlantic. Retrieved from https://www.theatlantic.com/technology/archive/2014/05/in-search-of-the-protomemex/371385/ 


\section{Endnotes}

1. See Barnett in this volume on the role of links and types of linking in Doug Engelbart's oN-Line System (NLS) and Ted Nelson's Xanadu in more detail.

2. Berners-Lee's first website at CERN also contained two directories of websites, one organized by subject and the other by type of service, see:

\section{http://info.cern.ch/hypertext/DataSources/Top.html.}

3. See danah boyd's (2006) critique of defining blogging as a form or genre, instead, she argues that it should be seen as both a medium and a practice.

4. As well as users from web fora, message boards and other early forms of web-based communication groups ( $\mathrm{xx}$, in this volume).

5. See Helmond (2013) on the history of social buttons and the technical details of link automation.

6. Which elements are visible in the preview depends on whether or not the website with the Share button has optimized itself for Facebook's crawler by employing Open Graph tags, Facebook’s markup language.

7. See Helmond (2013) on the history of link shorteners, the technical details behind link shortening and the role of shortened URLs in social media.

8. Technically this means that the browser displays the long URL, whilst the short URL is coded into the underlying HTML.

9. For example, Apple's Universal Links only work with iOS 9 or higher and not with Android apps, thereby only interlinking Apple apps into an ecosystem. 